\title{
SPIN PHYSICS PROGRESS WITH THE STAR DETECTOR AT RHIC
}

\author{
J. SOWINSKI \\ Indiana University Cyclotron Facility, \\ 2401 Milo B. Sampson Ln., \\ Bloomington, IN 47408, USA \\ E-mail: sowinski@iucf.indiana.edu \\ FOR THE STAR COLLABORATION
}

Progress in STAR over the last two years and projections for the coming two years are discussed. Important technical developments have been the completion of the barrel and endcap electromagnetic calorimeters. Measurement of inclusive jet and $\pi_{0} \mathrm{~A}_{L L}$ over the next two years are expected to answer whether $\Delta \mathrm{G}$ is a major contributor to the proton's spin. Transverse effects such as Collins and Sivers functions at mid-rapidity will also be investigated.

Since the first data from polarized proton collisions at RHIC were presented $^{1}$ at SPIN 2002 the STAR collaboration has continued to participate in the short data acquisition periods provided as the beams were developed in 2003 and 2004. During these periods, characterized by increasing luminosity and beam polarization, STAR has been able to commission new detectors important to its spin program as well as take data that will help tune the detectors for the upcoming runs and provide "first looks" with limited statistics at spin observables necessary to understanding the spin structure of the nucleon. With luminosity and polarization continuing to improve ${ }^{2}$, it is expected that extended running periods for polarized protons will be provided over the next few years allowing significant constraints to be placed on $\Delta \mathrm{G}$ and investigations of transverse physics such as Sivers and Collins functions to be started. In the following I will review STAR's upgrades of the past few years, the measurements already made and prospects for the next few years leading to SPIN 2006.

The STAR detector ${ }^{3}$ is based on a large solenoidal magnet with much of its internal volume devoted to tracking charge particles with a time projection chamber(TPC) in a $5 \mathrm{kG}$ magnetic field. A big effort has gone into pro- 
viding electromagnetic calorimetry around the outer diameter of the TPC, the barrel electromagnetic calorimeter (BEMC) $)^{4}$, and covering one poletip of the magnet, the endcap electromagnetic calorimeter $(\text { EEMC })^{5}$. Since 2002 subsets of these calorimeters have been installed and commissioned, with the installation of the final modules and electronics taking place in preparation for the 2005 run. This completes STAR's large solid angle capabilities allowing for the full reconstruction of jets (unique at RHIC) in addition to providing fast triggers, both essential to the spin program.

The two calorimeters are both lead/plastic-scintillator sampling calorimeters capable of measuring gamma rays from below $1 \mathrm{GeV}$ to over $100 \mathrm{GeV}$ in energy. The BEMC covers the range in pseudorapidity, $\eta=-\ln (\tan (\theta / 2))$, of $-1 \leq \eta \leq 1\left(\theta \geq 40^{\circ}\right)$ with 4800 individually read out "tower" energy signals. The EEMC provides an additional 720 tower energy signals in a forward pseudorapidity range, $1.1 \leq \eta \leq 2\left(37^{\circ} \geq \theta \geq 15^{\circ}\right)$, important for reaching small $\mathrm{x}_{\text {gluon }}$ in partonic collisions. The first two active layers of each detector are read out twice, once as part of the tower sum and independently as a pre-shower signal to help in $\pi^{0} / \gamma$ and electron/hadron discrimination. The last layer of the EEMC is also read out twice, again in the tower sum and independently as a post-shower signal for $\mathrm{e} / \mathrm{h}$ discrimination. Both detectors incorporate a finely segmented shower maximum detector (SMD) approx. 5 radiation lengths deep in the detector. The BEMC SMD is a gaseous proportional wire chamber while the EEMC uses triangular scintillator strips laminated into two crossed planes. These SMDs are used to reconstruct the 2 showers from $\pi^{0}$ decay vs. the single shower of direct photons. Although not complete over the full solid angle, the full functionality of these detector subsystems was used in the 2004 RHIC run. Currently algorithms such as those for finding $\pi^{0} \mathrm{~s}$ and jets are being fine tuned and physics analyses have begun.

It is well known that the spin of the quarks do not account for the spin of a proton ${ }^{6}$. Other contributions such as the spin of gluons $(\Delta \mathrm{G})$ or the orbital angular momentum are poorly constrained by existing data. Partonic scattering processes in $\vec{p}-\vec{p}$ scattering such as $\mathrm{q}+\mathrm{g} \rightarrow \mathrm{q}+\mathrm{g}$, $\mathrm{g}+\mathrm{g} \rightarrow \mathrm{g}+\mathrm{g}$ and $\mathrm{q}+\mathrm{g} \rightarrow \mathrm{q}+\gamma$ detected as jets, and in the last case an isolated direct photon, have large spin sensitivities ${ }^{6}$, opening a window to $\Delta \mathrm{G}$. The large solid angle of STAR is particularly well suited for detecting jets and direct-photon/jet coincidences over a range in pseudorapidity that helps cover a broad range in the partonic momentum fraction $\mathrm{x}$.

A long term goal for the STAR spin physics program is to measure $\Delta \mathrm{g}(\mathrm{x})$, i.e. not just the integral $\Delta \mathrm{G}$ over some region but the $\mathrm{x}$ dependence 


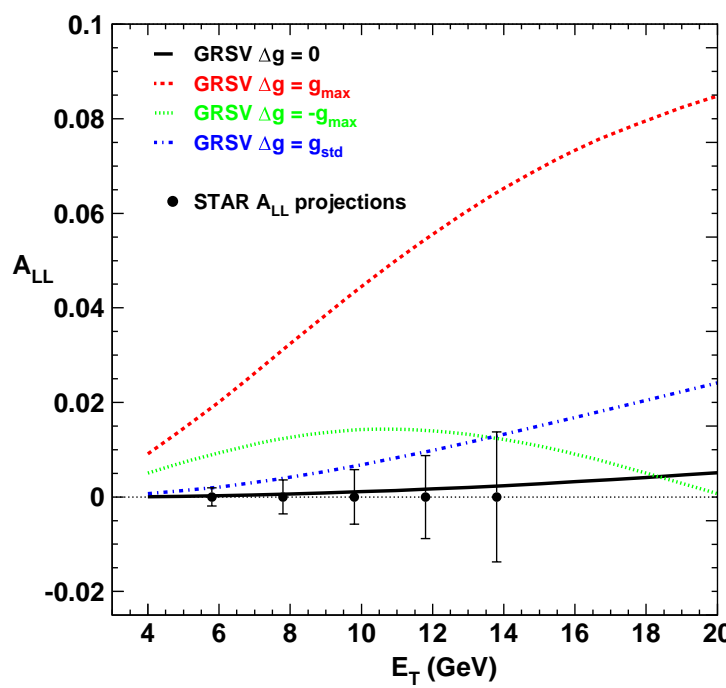

Figure 1. Predictions of $\mathrm{A}_{L L}$ for inclusive jets ${ }^{7}$ compared with the precision of measurements from data expected in the 2005 running period. An integrated luminosity of $7 \mathrm{pb}^{-1}$ with a polarization of 0.4 as well as jet finding efficiencies from previous runs is assumed.

from $0.01 \leq \mathrm{x} \leq 0.3$, using the $\mathrm{q}+\mathrm{g} \rightarrow \mathrm{q}+\gamma$ (QCD Compton scattering) process. The coincident direct-photon and jet present a relatively clean experimental signal, the partonic subprocess dominates other partonic subprocesses generating the same signal and two body kinematics allows the reconstruction of the partons' $\mathrm{x}$ and scattering angle. However this is a rare

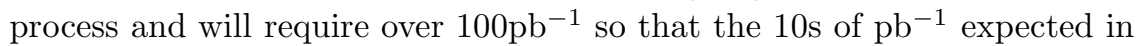
the next 2 runs will serve primarily to tune the technique.

Although not as selective, $A_{L L}$ measured with inclusive jets is sensitive ${ }^{7}$ to $\Delta \mathrm{G}$ as shown in Fig. 1, and jets are produced abundantly in the collisions at RHIC. The STAR detector has a large enough acceptance to reconstruct jets, and jet finding algorithms are being tuned on the 2004 data set. STAR triggers on jets either with a high tower trigger (largest tower above a threshold) or jet patch triggers (one of a predefined set of regions approx. $1 \times 1$ in $\eta \times \phi$ exceed a threshold). These triggers were tested and used during the 2004 run and preliminary analyses indicate that approximately $1 / 2$ of such triggers are found to contain jets with $\mathrm{p}_{T}$ over $5 \mathrm{GeV}$. It is expected that when fully analyzed the 2004 data will only be able to discriminate between the maximal $\Delta \mathrm{G}$ and 0 . The level of sensitivity expected with 2005 data is displayed in Fig. 1. The calorimeters in STAR are also capable of 
reconstructing $\pi^{0} \mathrm{~s}$. Jäger et al. have shown ${ }^{7}$ that jet sensitivity to $\Delta \mathrm{G}$ is carried over to the inclusive $\pi^{0} \mathrm{~A}_{L L}$. We expect that our inclusive $\pi^{0}$ results will have similar discriminating power to that of the jets but with different sensitivities to trigger bias.

A number of mechanisms have been suggested for explaining the forward $\pi^{0}$ analyzing powers we have measured ${ }^{1,8}$. STAR is also interested in pursuing effects arising from these at midrapidity. If the asymmetries in jet decays reflecting the showering quark's spin, as represented by Collins functions ${ }^{9}$, turn out to be large enough, they can be used to gain access to transversity via spin transfer from the final to initial state quarks. Boer and Vogelsang have also recently pointed out possible effects at a measurable level resulting from gluonic Sivers functions in dijets. ${ }^{10}$ The Sivers functions describe a correlation between the $\mathrm{k}_{T}(\mathrm{x})$ of partons and the transverse spin of the proton. While these correlations integrate ${ }^{11}$ to 0 over all partons and Bjorken $\mathrm{x}$, they need not be zero for individual partons. STAR has already measured the unpolarized $\mathrm{k}_{T}$ distribution ${ }^{12}$ and it is of sufficient width that, with transverse data expected in the next few years, we should be able to observe signals of the predicted size. ${ }^{10}$

Improving polarization and luminosity as well as significant running time for polarized protons at $\sqrt{s}=200 \mathrm{GeV}$ is expected over the next few years. In addition to continuing our studies of transverse physics, we hope that by spin 2006 we will be able to tell you whether gluons are a major contributor to the proton's spin or not.

\section{References}

1. G. Rakness in Spin 2002: 15th Int'l Spin Physics Symposium, eds. Y.I. Makdisi, A.U. Luccio and W.W. McKay, (AIP, New York, 2003) p. 400; J. Adams et al., Phys. Rev. Lett. 93 (2004) 012301.

2. W.W. MacKay, 'Status of Proton Polarization at RHIC and AGS', these proceedings.

3. K.H. Ackermann et al., Nucl. Instrum. Meth. A 499, 624 (2003).

4. M. Beddo et al., Nucl. Instrum. Meth. A 499, 725 (2003).

5. C.E. Allgower et al., Nucl. Instrum. Meth. A 499, 740 (2003).

6. G. Bunce et al., Ann. Rev. Nucl. Part. Sci., 525 (2000).

7. B. Jager, M. Stratmann, W. Vogelsang, Phys.Rev. D70, 034010 (2004).

8. A. Ogowa, Spin Physics in Large Rapidity Neutral Pion Production at STAR, these proceedings.

9. J.C. Collins, S.F. Heppelmann, G.A. Ladinsky, Nucl. Phys. B420, 565 (1994).

10. D. Boer, W. Vogelsang, Phys.Rev. D69, 094025 (2004).

11. M. Burkardt, Phys.Rev. D69, 091501 (2004).

12. T. Henry, J.Phys. G30 S1287 (2004). 